with learning curve and success rate improved during the study period.

Disclosures E. Almallouhi: None. S. Al kasab: None. S. Pai: None. J. Lena: None. A. Spiotta: 1; C; Penumbra. 2; C; Penumbra, Stryker, Cerenovus, Terumo.

\section{E-170 BENIGN CEREBRAL VASOCONSTRICTION IMMEDIATELY AFTER CAROTID ARTERY STENTING}

D Park*, T Kim. Radiology, Hanyang University Guri Hospital, Guri, Korea, Republic of

10.1136/neurintsurg-2020-SNIS.202

Purpose This study is to evaluate benign cerebral vasoconstriction on the ipsilateral anterior circulation immediately after carotid artery stenting (CAS) in the near-occlusion of proximal internal carotid artery (ICA).

Materials and Methods 15 patients with near occlusion were retrospectively reviewed, who were selected among all patients who conducted CAS for proximal ICA stenosis since January 2009. Immediate post-stenting angiographic images were evaluated by two neuroradiologists for the presence of vasoconstriction.

Results Among 15 patients with near-occlusion, significant vasoconstrictions in the ipsilateral anterior circulation after CAS were shown in 7 patients $(71.1 \pm 5.3$ years $)$ but not in the other 8 patients. In all 7 patients, significant vasoconstrictions were shown to occur in isolated territory without anterior and/or posterior communicating collateral flows $(\mathrm{P}<0.001)$. However, there are no significant statistical differences in neurologic status, procedural conditions, and comorbidity between two groups.

Conclusions Benign cerebral vasoconstriction immediately after carotid artery stenting could occur in the ipsilateral anterior circulation and in the condition with near-occlusion and isolated circulation.

Disclosures D. Park: None. T. Kim: None.

\section{E-171 THE BASILAR SHIELD: A NORMAL VARIANT BULBOUS CONFIGURATION OF THE BASILAR ARTERY TIP}

K McElroy*, W Brinjiki. Radiology, Mayo Clinic, Rochester, MN

\subsection{6/neurintsurg-2020-SNIS.203}

Introduction The intracranial arterial vasculature has numerous anatomical variants, which vary from largely benign to having remarkable clinical significance. A lesser known variant can be described as a bulbous configuration of the basilar artery tip, also referred to as a 'shield-like appearance' or 'junctional dilatation.' However, this variant's significance and standard nomenclature have not been described in the literature to as of yet.

Methods Retrospective query of radiology imaging reports was performed for vascular specific imaging of the head (CTA/ MRA/cerebral angiography), which included descriptions related to the basilar artery anatomical variant from 20052019 at a single institution. Key-word search descriptions included 'bulbous tip', 'junctional dilatation', and 'shield-like' in addition to 'basilar.' Imaging studies were individually scrutinized for true presence of the basilar variant, and were assessed for any change in appearance over time. Primary data-points collected included length of time between imaging studies with stable imaging appearance, basilar artery aneurysm incidence, other intracranial aneurysm incidence, and frequency of subarachnoid hemorrhage associated with aneurysm rupture.

Results Initial query yielded 345 imaging reports which included the basilar artery search terms in question. Fiftytwo patients were found to have multiple vascular exams of the head on different occasions, and 50 of these were confirmed to have the typical appearance of the basilar shield variant present on imaging. Risks factors of cerebral aneurysm development were variably present in this cohort, including hypertension, history of smoking, family history of cerebral aneurysms, connective tissue disease, and autosomal dominant polycystic kidney disease. On review of follow-up imaging studies, the basilar shield variant was found to be stable in appearance over time in all 50 patients, without interval expansion or basilar tip aneurysm development. Interval follow-up ranged from 39 to 4,664 days, with a mean follow-up length of 3.83 years, and total patient-years of follow-up of 192. More than half $(54 \%)$ of this patient cohort demonstrated concurrent cerebral arterial aneurysms. Three patients were found to have history of subarachnoid hemorrhage related to cerebral aneurysm rupture, however none of these cases were related to the basilar artery. No adverse neurological events were found which could be attributed to the basilar shield variant.

Conclusion The bulbous configuration of the basilar artery tip dubbed the 'basilar shield' is a benign, normal variant of the posterior intracranial arterial circulation. Further studies may be necessary to determine if there is any association with cerebral aneurysms, but the variant itself is innocuous. It is important for both radiologists and clinicians to recognize its characteristic appearance and benign nature. True basilar tip aneurysms have a high morbidity and mortality in rupture, and confusion with the basilar shield may lead to unnecessary work-up, imaging, and/or treatment.

Disclosures K. McElroy: None. W. Brinjiki: None.

\section{E-172 CT PERFUSION DERIVED HYPOPERFUSION INTENSITY RATIO PREDICTS INFARCT VOLUME AND GROWTH PRE- AND POST-ENDOVASCULAR THERAPY IN LARGE VESSEL OCCLUSION ACUTE ISCHEMIC STROKE}

${ }^{1} \mathrm{M}$ Jutras*, ${ }^{2} \mathrm{D}$ Lyndon, ${ }^{2} \mathrm{~B}$ Niu, ${ }^{2} \mathrm{M}$ Lloret Villas, ${ }^{1} \mathrm{~S}$ Yip, ${ }^{1} \mathrm{~F}$ Settecase. ${ }^{1}$ University of British Columbia, Vancouver, BC, CANADA; ${ }^{2}$ Vancouver General Hospital, Vancouver, BC, CANADA

\subsection{6/neurintsurg-2020-SNIS.204}

Purpose Evaluation of large vessel occlusion (LVO) acute ischemic stroke (AIS) patients for endovascular therapy (EVT) is a multifaceted decision-making process. Previous work using CT angiography has demonstrated that collateral blood supply is a key determinant of infarct volume and functional outcomes in LVO AIS. The CT Perfusion (CTP) derived Hypoperfusion Intensity Ratio (HIR), Tmax $>10$ s over Tmax $>6 \mathrm{~s}$ within the affected territory, is a surrogate marker of collateral status. The purpose of this study is to investigate whether an association exists between HIR and infarct volumes in LVO AIS.

Materials and Methods IRB approval was obtained. The medical records and imaging of patients with suspected LVO AIS 

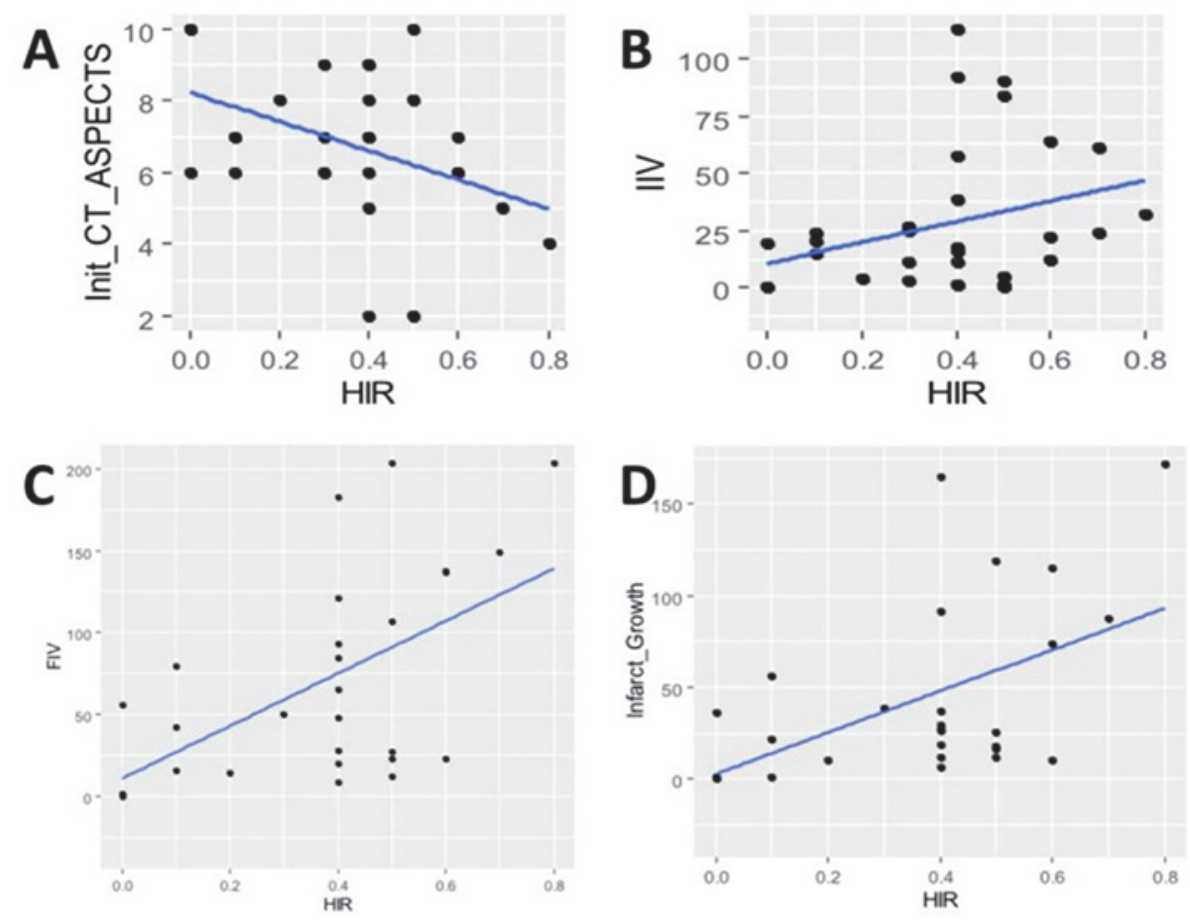

\section{Abstract E-172 Figure 1}

and undergoing CTP (between 0-24 hours since last known well) and treated with EVT between March to September 2019 were retrospectively reviewed. Patients with preexisting infarcts were excluded. CTP was processed using software (RAPID, Ischemaview) with HIR automatically calculated. ASPECTS scores were obtained and infarct volumes calculated using the $\mathrm{ABC} / 2$ method on the initial noncontrast $\mathrm{CT}$ and CT performed 24-72 hours post-EVT. Spearman correlation test were performed between HIR and initial CT ASPECTS, initial CT infarct volume. After excluding 5 patients without successful reperfusion, HIR Spearman correlation with postEVT CT ASPECTS, final infarct size, and infarct growth was assessed.

Results Thirty-two patients were included in the study (20 males and 12 females), with a mean age of 63.0 years $(\mathrm{SD}=17.3)$. Initial ASPECTS scores ranged from 2 to 10 (median 7). Initial infarct volume (IIV) ranged from 0 to 113 $\mathrm{ml}$ (median 19). Successful reperfusion (mTICI 2B, 2C, or 3) was obtained in 28/32 (88\%). Final ASPECTS scores at discharge ranged from 0 to 10 (median 5.5), and final infarct volume (FIV) ranged from 0 to $367 \mathrm{ml}$ (median $72 \mathrm{ml}$ ). There was a significant correlation between HIR and initial ASPECTS (-0.37, $\mathrm{p}=0.03)$, and a trend to significance with IIV $(0.32, \mathrm{p}=0.07)$ (figure $1 \mathrm{~A}$ and $1 \mathrm{~B})$. With non-recanalizers excluded, a significant correlation was observed between HIR and final ASPECTS (-0.51, p=0.006), FIV (0.50, p=0.009), and infarct growth pre- and post-EVT (0.41, $\mathrm{p}=0.03$ ) (figure $1 \mathrm{~B}$ and $1 \mathrm{C})$.

Conclusions In the time-sensitive environment of LVO AIS, HIR allows rapid assessment of collateral status, showing similar correlations with infarct size as CTA collateral scoring. Further investigation is warranted to determine whether HIR is predictive of clinical outcomes.

Disclosures M. Jutras: None. D. Lyndon: None. B. Niu: None. M. Lloret Villas: None. S. Yip: None. F. Settecase: None.

\section{E-173 ASSESSING AGE RELATED DISCREPANCIES OF TOTAL CEREBRAL BLOOD FLOW AND CEREBRAL VENOUS OUTFLOW USING 4D FLOW MRI}

${ }^{1} \mathrm{R}$ Abdalla*, 'S Schnell, ${ }^{1} \mathrm{M}$ Aristova, ${ }^{2} \mathrm{M}$ Hurley, ${ }^{3} \mathrm{~S}$ Ansari, ${ }^{2} \mathrm{~A}$ Shaibani. ${ }^{1}$ Radiology, Northwestern University - Feinberg school of Medicine, Chicago, IL; ${ }^{2}$ Radiology, Neurosurgery, Northwestern University - Feinberg school of Medicine, Chicago, IL; ${ }^{3}$ Radiology, Neurosurgery and Neurology, Northwestern University - Feinberg school of Medicine, Chicago, IL

\subsection{6/neurintsurg-2020-SNIS.205}

Purpose The cerebral arterial inflow, venous outflow, and Cerebrospinal fluid volume and flow are highly regulated in an equilibrium to maintain adequate cerebral perfusion as governed by the Monro-Kellie doctrine. Multiple neurological conditions had been associated with abnormalities in the cerebral venous system including multiple sclerosis and idiopathic intracranial hypertension, among others. We aim to quantitatively study the relationship and discrepancy between total cerebral blood flow (TCBF) and cerebral venous outflow (CVO), as well as the TCBF as a proportion of the cardiac output in adults and how it compares to the pediatric patients, and highlight the discrepancies.

Materials and Methods 31 healthy volunteers (24 adults and 7 children) underwent 4D flow MR imaging for arterial evaluation and 2D CINE PC-MRI for venous evaluation in an IRB approved study. 3D volumetric ECG-gated PC-MRI data with 3-directional velocity encoding (4D flow MRI) was acquired for the arterial system. 2D CINE PC MRI was acquired for the venous system at both transverse sinuses. 4D flow MRI data was processed using in-house software tools in Matlab. We calculated the cumulative TCBF in $\mathrm{ml} / \mathrm{sec}$ as the sum of inflow in the bilateral ICAs and basilar artery. The CVO was calculated as the sum of the flow in the bilateral transverse sinuses. The ratio of $\mathrm{CVO}$ to TCBF was calculated. Also, the TCBF as a percentage of cardiac output was calculated. 